\title{
ON SIMPLICES AND LATTICE POINTS
}

\author{
P. R. SCOTT
}

(Received 21 March 1980)

Communicated by A. J. van der Poorten

\begin{abstract}
Let $S$ be a simplex in $E^{n}$ which is homothetic to a given simplex $S^{*}$, which contains no point of the integral lattice in its interior, and which has maximal volume $V(S)$. We conjecture that $V(S)>$ $n^{n} / n$ !, and establish the conjecture for $n<3$.

1980 Mathematics subject classification (Amer. Math. Soc.): primary 52 A 20, 52 A 43; secondary $10 \mathrm{E} 05$.
\end{abstract}

\section{Introduction}

The problem considered here is analogous to a problem proposed by Mordell [2], in which a lower bound is sought for the volumes of certain specified parallelopipeds centred at the origin. Mordell's conjecture is established for $n=2,3$ by Szekeres [4], [5] and Ko [1].

Two simplices in euclidean $n$-space, $E^{n}$, are said to be homothetic if they are similar and similarly placed. Let $S^{*}$ be any given simplex. We say that $S$ is a maximal simplex if $S$ is homothetic to $S^{*}, S$ contains no points of the integral lattice $\Gamma_{n}$ in its interior, and $V(S)$ is maximal.

CONJECTURE. If $S$ is a maximal simplex in $E^{n}$, then

$$
V(S)>n^{n} / n !
$$

and this lower bound is best possible.

OCopyright Australian Mathematical Society 1981 
We notice that this bound is attained for the simplex with vertices $0,(n, 0, \ldots, 0), \ldots,(0, \ldots, 0, n)$. Since the interior of this simplex is defined by $x_{i}>0(1 \leqslant i \leqslant n), \sum_{i=1}^{n} x_{i}<n$, it is clear that no point of $\Gamma_{n}$ lies in the interior.

The conjecture is trivially true for $n=1$. We shall establish it for $n=2,3$.

\section{The question of existence}

Let $S$ be a maximal simplex in $E^{n}$. We ask if there exists a real positive number $\kappa_{n}$ such that $V(S) \geqslant \kappa_{n}$. If the existence of $\kappa_{n}$ can be established for each $n$, then the conjecture is easily proved. We show here that $\kappa_{2}, \kappa_{3}$ exist. However, as in Mordell's problem, for $n>3$ the problem of existence seems to be intractable.

If $S$ is a maximal simplex homothetic to a given simplex $S^{*}$, then clearly each $(n-1)$-dimensional face of $S$ must contain a lattice point in its relative interior. For if not, the face can be translated outwards without introducing any lattice points into the interior of $S$, and $S$ is not maximal.

The Case $n=2$. Let $A, B, C$ be lattice points on the edges of a maximal triangle $S$. Since $A, B, C$ cannot be collinear, they form the vertices of a lattice triangle which has area not less than $\frac{1}{2}$, and which is contained in $S$. Hence $V(S)>\frac{1}{2}$, and we may take $\kappa_{2}=\frac{1}{2}$.

The Case $n=3$. Let $A, B, C, D$ be lattice points in the relative interiors of the faces of a maximal tetrahedron $S$. If $A, B, C, D$ are not coplanar, then they form the vertices of a lattice tetrahedron of volume not less than $\frac{1}{6}$, and we obtain $V(S) \geqslant \frac{1}{6}$.

Suppose then that $A, B, C, D$ are coplanar. By suitable integral unimodular transformation of $S$ (and $S^{*}$ ) we may assume these points lie in the $x y$-plane. Now $S$ cannot lie in the region $|z|<\frac{1}{2}$, for then we could obtain a homothetic simplex $S^{\prime}$ of larger volume and containing no lattice points in its interior by translating $S$ into the region $0<z<1$ and then enlarging. Hence we may assume without loss of generality that there is a point $P$ of $S$ in the plane $z=\frac{1}{2}$. Since $S$ is convex, points $A, B, C$ are not collinear, and $A, B, C, P$ form the vertices of a proper tetrahedron contained in $S$. As the volume of this tetrahedron is not less than $\frac{1}{12}$, we have $V(S)>\frac{1}{12}$.

Hence we may take $\kappa_{3}=\frac{1}{12}$. Alternatively, we can obtain $\kappa_{3}>\frac{1}{3}$ by suitably dissecting the parallelopiped considered by Szekeres [5]. 


\section{Two preliminary lemmas}

We shall need the following two results.

LEMMA 1. If the centre of gravity of each face of a simplex $S$ in $E^{n}$ is a point of $\Gamma_{n}$, then so is each vertex of $S$. Further, $V(S)>n^{n} / n$ !.

Proof. Let $v_{1}, v_{2}, \ldots, v_{n+1}$ denote the vertices of $S$, and let $c_{i}$ denote the centre of gravity of the $(n-1)$-dimensional face not containing $v_{i}(1<i<$ $n+1)$.

Then

$$
n \mathbf{c}_{i}=\sum_{k=1}^{n+1} \mathbf{v}_{k}-\mathbf{v}_{i} \quad(1<i<n+1) .
$$

Solving these equations for $\mathbf{v}_{i}$ gives

$$
\mathbf{v}_{i}=\sum_{k=1}^{n+1} \mathbf{c}_{k}-n \mathbf{c}_{i} \quad(1<i \leqslant n+1) .
$$

Hence if $c_{1}, \ldots, c_{n+1}$ are points of $\Gamma_{n}$, then so are the vertices $v_{1}, \ldots, v_{n+1}$. Further,

$$
\mathbf{v}_{i}-\mathbf{v}_{j}=-n\left(\mathbf{c}_{i}-\mathbf{c}_{j}\right)
$$

Hence the simplex $S$ is homothetic to the lattice simplex $L$ having vertices $c_{1}, \ldots, c_{n+1}$, and $n$ times as large. Since $V(L)>1 / n$ !, we deduce that $V(S)>$ $n^{n} / n !$.

LEMMA 2. Let $T$ be an $(n-1)$-dimensional simplex in $E^{n}$ having centre of gravity t. Let $F$ be an $(n-2)$-dimensional flat intersecting $T$ and separating it into subsets $U, W$. Let $T$ be rotated about $F$. Then

(i) $U, W$ sweep out equal volumes if and only if $\mathbf{t}$ lies in $F$;

(ii) $U$ sweeps out a larger volume than $W$ if and only if $F$ strictly separates $t$ from $W$ (in the hyperplane of $T$ ).

Proof. (i) Let $U, W$ have centres of gravity $\mathbf{u}, \mathbf{w}$ respectively, and let $|U|,|W|$ denote the $(n-1)$-dimensional volumes of $U, W$. Without loss of generality, let the reference system be chosen such that

(a) the distance of each point $\mathrm{x}=\left(x_{1}, x_{2}, \ldots, x_{n}\right)$ of $T$ from $F$ is given by $\left|x_{1}\right|$

(b) if $\mathbf{x} \in U, x_{1}>0$, and if $\mathbf{x} \in W, x_{1}<0$. Now since $\mathbf{t}$ is the centre of gravity of $T$,

$$
(|U|+|W|) \cdot t_{1}=|U| \cdot u_{1}+|W| \cdot w_{1}
$$


Also, according to the extended version of Pappus' Theorem (see for example [3]), the volume of revolution generated by $U$ is given by the product of $|U|$ and the length of the path of the centre of gravity $\mathbf{u}$. Hence if $T$ is rotated about $F$ through angle $\theta(>0)$, the volume generated by $U$ is $|U| \cdot u_{1} \cdot \theta$. Similarly, noting that $w_{1}<0$, the volume generated by $W$ is $|W| \cdot\left(-w_{1}\right) \cdot \theta$. These generated volumes are equal if and only if

$$
|U| \cdot u_{1}+|W| \cdot w_{1}=0 .
$$

But this is precisely the condition for $t$ to lie on $F$.

(ii) Suppose now that $|U| \cdot u_{1} \cdot \theta>|W| \cdot\left(-w_{1}\right) \cdot \theta$. This occurs when and only when $|U| \cdot u_{1}+|W| \cdot w_{1}>0$. But this is the condition that $t_{1}>0$, and $F$ strictly separates $\mathbf{t}$ from $W$ as required.

\section{Proof of the conjecture, assuming existence}

Let us assume the existence of a maximal simplex $S$ of smallest volume in $E^{n}$. We have seen that each $(n-1)$-dimensional face $T$ of $S$ contains at least one interior lattice point. Suppose $T$ contains a lattice point in its relative interior, but not at its centre of gravity $t$. Then we can choose a lattice point $p$ interior to $T$, and an $(n-2)$-dimensional face $R$ of $T$, such that $\mathrm{p}$ is closer to $R$ than $\mathrm{t}$, and at least as close to $R$ as any other lattice point interior to $T$. Choose an $(n-2)$-dimensional axis (flat) $F$ through $\mathbf{p}$ and parallel to $R$. Now there exists a small rotation of $T$ about $F$ which introduces no new lattice points into the interior of $S$, and which by Lemma 2 decreases the volume of $S$. Hence $S$ does not have smallest volume.

We deduce that each face of $S$ contains just one lattice point at its centre of gravity. But now by Lemma 1 , each vertex of $S$ is a lattice point, and $V(S) \geqslant n^{n} / n !$.

Thus the conjecture has been established, providing a maximal simplex of smallest volume exists in $E^{n}$. It is certainly true for $n \leqslant 3$.

\section{References}

[1] C. Ko, 'Note on the lattice points in a parallelopiped', J. London Math. Soc. 12 (1936), 40-47.

[2] L. J. Mordell, 'Note on an arithmetical problem on linear forms', J. London Math. Sac. 12 (1936), 34-36.

[3] D. M. Y. Sommerville, An introduction to the geometry of $n$ dimensions (Methuen, London, 1929). 
[4] G. Szekeres, 'On a problem of the lattice plane', J. London Math. Soc. 12 (1937), 88-93.

[5] G. Szekeres, 'Note on lattice points within a parallelopiped', J. London Math. Soc. 12 (1937), 36-39.

Department of Pure Mathematics

University of Adelaide

Adelaide

South Australia 